\title{
Relação entre a velocidade, a agilidade e a potência muscular de futebolistas profissionais
}

\author{
António N. Rebelo \\ José Oliveira
}

RESUMO

A velocidade, agilidade e a potência muscular são qualidades importantes para a performance física do futebolista. No pre sente estudo, avaliou-se o desempenho de futebolistas em testes de terreno e de laboratório para as referidas capacidades e determinou-se a força de associação entre elas.

Foram avaliados 23 futebolistas profissionais participantes na Super Liga de Futebol Portuguesa, aos quais foram aplicados os seguintes testes: velocidade em $15 \mathrm{~m}$ (V15), velocidade em $35 \mathrm{~m}$ (V35), agilidade ( $A G I)$ e potência máxima(MP) em dinamómetro isocinético (Biodex - System 3, NY, USA).

A força de associação entre as medidas dos testes V15, V35 e AG revel ou ser moderadamente elevada a elevada $(r=0,60$ a $r=0,86)$. A MP dos músculos extensores e flexores do joelho mostrou uma correlação moderada com a performance no V15 e no AGI, mas

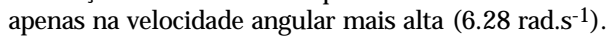

Os resultados do presente trabal ho sugerem que a MP é fundamental para o desempenho nos testes V15 e AGI. A determinação da MP em dinamómetro isocinético deve ser obtida em velocidades angulares tão elevadas quanto o instrumento o possibilite. Finalmente, embora os testes não se substituam uns aos outros, os testes V15 e AGI estarão mais próximos nos atributos que cada um deles avalia.

Palavras-chave: futebol, velocidade, agilidade, potência muscular

\author{
Faculdade de Desporto \\ U niversidade do Porto \\ Portugal
}

\section{ABSTRACT \\ Association between speed, agility and muscular power of pro soccer players}

Speed, agility and muscle power are important physical abilities for soccer player performance. The aim of this study was to evaluate the above-mentioned abilities and to determine the power of association between them, using the results obtained by soccer players in field and laboratory tests

Twenty three professional soccer players participating in Portuguese First League were evaluated in the following tests: $15 \mathrm{~m}$ sprint test (V15), 35m sprint test (V35), agility (AGI) and isokinetic strength test (Biodex - System 3, NY, USA) from which maximal power (MP) was estimated.

The power of association between V15, V35 and AGI was moderate high to high ( $r=0,60$ to $r=0,86)$. MP of knee extensors and flexors muscles was found to correlate moderately with the performance in V15 and AGI, but only at higher angular velocities ( $6.28 \mathrm{rad}^{-1} \mathrm{~s}^{-1}$. The results of the present study suggest that MP has an important contribution to V15 and AGI performance. MP evaluation in isokinetic dynamometers must be determined at high angular velocities. Finally, although the tests couldn't be used interchangeably, V15 and A GI seem to have many attributes in common.

Key-words: soccer, speed, agility, muscle power 


\section{INTRODUÇÃO}

A velocidade, a agilidade e a potência muscular são profusamente citadas na literatura como componentes importantes da performance física de um futebolista. Com efeito, num determinado momento do jogo, ser mais rápido permitirá chegar primeiro, ser mais ágil evitará o iminente impacto com um adversário e ser mais potente contribui para o sucesso do jogador em ambas as acções. Dito por outras palavras, se bem que seja uma evidência, que não é melhor futebolista aquele que é mais rápido, ágil ou potente, será também de todo irresponsável e leviano pensar-se que estes atributos físicos, desenvolvidos à luz de princípios adequados do treino e das exigências funcionais específicas do jogo de futebol, não contribuem para que o jogador e a sua equipa sejam mais capazes (para ref.'s ver 1).

A característica da velocidade de deslocamento que mais interessa para o futebolista diz respeito à fase de aceleração, com e sem mudança de direcção, em conjugação com a agilidade e não à velocidade máxima (5). Estudos do tipo tempo e movimento mostraram que os esforços de intensidade máxima realizados pelos futebolistas em jogo se caracterizam por serem de curta duração ( $2-6$ segundos) e que os deslocamentos em sprinte se associam, muitas vezes, a mudanças de direcção e/ ou de sentido da corrida e a travagens bruscas (cerca de 25-30 vezes por jogo (12)), isto é, requerendo agilidade o que em última instância significa a adaptação e coordenação no deslocamento com o mínimo prejuízo da rapidez da acção.

Velocidade e agilidade são duas qualidades da performance física que exigem aval iação específica $(11,20)$. A velocidade e a agilidade são, habitual mente, avaliadas sobre distâncias curtas ( $5 \mathrm{~m}$ a $20 \mathrm{~m}$ ).

Diversas acções de jogo exigem a produção de níveis elevados de potência muscular. Destaque-se de entre essas acções os sprintes com e sem mudanças de direcção e/ ou sentido da corrida. Quando um atleta acelera ou desacelera de forma muito brusca são-lhe exigidos el evados níveis de força e potência para modificar a inércia da sua massa corporal. Por isso, quando se fala de velocidade e de agilidade no futebol, não deverá ser negligenciada a sua dependência da potência muscular $(15,19)$.

Pelo já exposto, parece ser de aceitar a ideia de que qualquer futebolista não recusaria a gentileza da natureza Ihe "oferecer" geneticamente a possibilidade de ter, simultaneamente, mais força, mais velocidade e mais agilidade. A este propósito, ainda hoje uma questão é colocada: estas três formas de manifestação da performance física estabelecem entre si al guma associação? Numa primeira análise, a resposta a esta questão deveria ser afirmativa.

São escassos os trabal hos em que se procurou relacionar as capacidades motoras velocidade, agilidade e força de futebolistas. Contudo, os trabalhos de (5, 10) descreveram uma fraca ou moderada associação entre a agilidade e a velocidade.

No presente estudo, pretendeu-se descrever a performance de velocidade, agilidade e de potência muscular de futebolistas de elite e testar a força de associação entre as medidas da performance nas capacidades anteriormente enunciadas.

\section{METODOLOGIA}

Amostra

A amostra deste estudo foi composta por 23 futebolistas profissionais participantes na Super Liga de Futebol Portuguesa (idade: $25.7 \pm 3.9$ anos; peso: $76.1 \pm 6.6 \mathrm{Kg}$; altura: $179.5 \pm 7.2 \mathrm{~cm}$; massa gorda: $8.2 \pm 7.2 \%)$.

O momento de aplicação dos testes na época desportiva situou-se na primeira semana do período preparatório.

A avaliação clínica dos atletas que fizeram parte da amostra não mostrou al terações da aptidão muscular resultantes de problemas clínicos prévios.

Instrumentos e procedimentos de avaliação Velocidade

A velocidade foi avaliada em linha recta nas distâncias de $15 \mathrm{~m}$ e de $35 \mathrm{~m}$.

A performance de velocidade foi expressa através do tempo gasto para percorrer as distâncias de $15 \mathrm{~m}$ e $35 \mathrm{~m}$, recorrendo-se para o efeito a 3 pares de células fotoeléctricas (Speed Trap II - Browser Timming Systems) colocadas no ponto de partida, aos $15 \mathrm{~m}$ e aos $35 \mathrm{~m}$. Os sprintes foram realizados a partir da posição de parado com o pé preferido $0.3 \mathrm{~m}$ atrás da linha de partida. O registo do tempo gasto em cada percurso foi anotado até aos centésimos de segundo. O teste foi realizado em superfície de relva natural seca e em boas condições de utilização, superfície uti- 
lizada pelos atletas nas suas rotinas de treino, tendo sido precedido por um aquecimento específico. Os atletas usaram como calçado, botas de futebol. Todos os atletas realizaram duas tentativas, tendo sido considerado, para estudo, o mel hor resultado.

A gilidade

A agilidade foi avaliada num teste de $20 \mathrm{~m}$ com mudanças sucessivas de direcção ao fim de cada fracção de $4 m$ (ver representação gráfica na Figural). Este tipo de teste exige acelerações e desacelerações e equilíbrio, que são facetas importantes da agilidade. Os futebolistas estavam familiarizados com este teste pelo que o efeito de aprendizagem terá sido negl igenciável. Na figura 1 é mostrado o esquema do teste de agilidade.

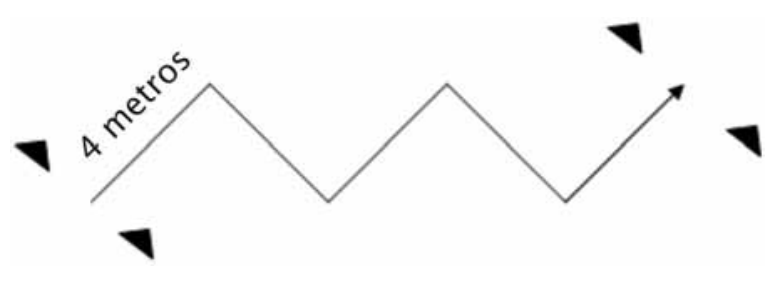

Figura 1. Teste de agilidade (adaptado de Little e Williams ${ }^{(10)}$ )

A performance de agilidade traduziu-se pelo tempo gasto para efectuar a distância do teste. $\mathrm{O}$ instrumento utilizado para o registo de tempos foi o mesmo que se descreveu para o teste de velocidade. No entanto, foram utilizados apenas dois pares de células, colocados aos $0 \mathrm{~m}$ e aos $20 \mathrm{~m}$.

No teste de agilidade, os atletas realizaram duas repetições com a primeira curva à esquerda e duas repetições com a primeira curva à direita. Foi considerado o melhor resultado de cada par de tentativas. As condições de realização do teste no que se refere à posição de partida, superfície e calçado foram similares às descritas para o teste de vel ocidade.

Avaliação da força e cálculo da potência

A força muscular dos músculos flexores e extensores do joel ho foi avaliada em dinamómetro isocinético (Biodex - System 3, NY, USA) com correcção gravítica dos torques máximos concêntricos, às velocidades angulares de $360^{\circ} . \mathrm{s}^{-1}$ (6.28 rad.s $\left.\mathrm{s}^{-1}\right)$ e $90^{\circ} . \mathrm{s}^{-1}$ (1.57 rad. $\mathrm{s}^{-1}$ ). Foram registados os torques máximos e o tempo gasto para o alcançar.

Os atletas realizaram um período de activação geral em cicloergómetro (M onark E-824, Vansbro, Suécia) com uma resistência correspondente a $2 \%$ do peso corporal durante um período de 5 minutos. Em seguida os sujeitos foram sentados na cadeira do dinamómetro e estabilizado o seu posicionamento com recurso a cintos colocados ao nível do tronco, do abdómen e da coxa, no sentido de prevenir movimentos acessórios. $\mathrm{O}$ joelho a avaliar foi posicionado a $90^{\circ}$ da flexão $\left(0^{\circ}=\right.$ extensão completa $)$ e o eixo de rotação do braço da alavanca do dinamómetro alinhado com a parte lateral do côndilo femoral.

Previamente à real ização do teste, procedeu-se à realização de 10 repetições sub-máximas de extensão/flexão do joel ho, para familiarização dos atletas ao dinamómetro, após o que se seguiu um período de recuperação de 1.5 minutos. Todos os sujeitos foram instruídos para colocarem de forma confortável os braços cruzados sobre o tronco tendo em vista o isolamento da acção dos grupos musculares responsáveis pela extensão e flexão do joelho. A avaliação no dinamómetro isocinético consistiu na realização de 5 repetições à velocidade angular de $360^{\circ} . \mathrm{s}^{-1}$ e de 3 repetições a $90^{\circ} . \mathrm{s}^{-1}$, por esta ordem e em cada um dos membros. Entre séries foi realizada uma pausa de repouso com a duração de 1.5 minutos. Os sujeitos foram instruídos para exercerem o máximo de força possível, tanto na extensão, como na flexão do joelho, sendo verbalmente encorajados. O cál culo da potência muscular foi efectuado a partir da seguinte equação (18):

Potência máxima (MP) = Torque máximo $(\mathrm{Nm}) \mathrm{x}$ velocidade angular ( rad. $^{-1}$ )

Procedimentos estatísticos

Para analisarmos os dados, com a finalidade de responder ao problema do estudo utilizaram-se os seguintes procedimentos:

Para a descrição dos dados recorreu-se aos procedimentos habituais da estatística descritiva, média, desvio padrão e amplitude.

Para estudar a associação entre as variáveis força, agilidade e velocidade recorreu-se à correlação de Pearson. O nível de significância foi estabelecido para $p \leq 0,05$. 


\section{RESULTADOS}

No Quadro 1 são apresentados os resultados obtidos pelos futebolistas nos testes de velocidade e de agilidade (tempo em segundos).

Quadro 1. Resultados dos futebolistas nos testes de velocidade e de agilidade.

\begin{tabular}{lcc}
\hline Testes & Média \pm SD $[\mathrm{s}$ ] & Amplitude $[\mathrm{s}$ ) \\
\hline V15 & $2.41 \pm 0.10$ & $2.21-2.60$ \\
V35 & $4.90 \pm 0.20$ & $4.46-5.39$ \\
AGI & $6.14 \pm 0.28$ & $5.58-5.68$ \\
\hline & s (segundos] &
\end{tabular}

Um dos factos mais relevantes a extrair do Quadro 1 são os elevados valores da amplitude dos resultados. No caso do teste de agilidade a amplitude é de 1 segundo.

Não foram encontradas diferenças significativas entre os valores médios do torque máximo dos membros dominante e não dominante.

No Quadro 2 são apresentados os valores da potência máxima (MP) dos músculos extensores e flexores do joel ho avaliados a duas velocidades angulares diferentes.

Quadro 2 - Valores (média \pm desvio-padrão) da potência máxima (média dos dois membros) dos músculos extensores e flexores do joelho nas duas velocidades avaliadas.

\begin{tabular}{|c|c|c|c|}
\hline$M P_{e x} t 1.57$ & $M P_{e x} t 6,28$ & $M P_{\text {flex }} 1.57$ & $M P_{\text {flex }} 6,28$ \\
\hline $355,1 \pm 32,2$ & $854,8 \pm 118,7$ & $125,7 \pm 14,9$ & $691,8 \pm 156,2$ \\
\hline
\end{tabular}

No Quadro 3 são apresentados apenas os valores das correlações entre as medidas das variáveis do estudo.

Quadro 3. Valores de correlação entre os diferentes testes.

\begin{tabular}{|c|c|c|c|c|c|c|c|}
\hline & V15 & V35 & AGI & $M P_{\text {ext }}$ & $M P_{\text {ext }}$ & $M P_{\text {Flex }}$ & $M P_{\text {Flex }}$ \\
\hline V15 & . & $0,73^{* *}$ & $0,86^{* *}$ & 0,31 & $0,44^{*}$ & 0,32 & $0,58^{* *}$ \\
\hline V35 & $0,73^{* *}$ & . & $0,60^{* *}$ & 0,06 & 0,11 & 0,29 & 0,32 \\
\hline AGI & $0,86^{* *}$ & $0,60^{* *}$ & . & 0,31 & $0,45^{*}$ & 0,23 & $0,62^{* *}$ \\
\hline
\end{tabular}

Como se pode observar não se registaram associações significativas entre os resultados do teste V35m e os valores da potência máxima dos músculos extensores eflexores do joelho, nas duas velocidades angulares consideradas.

Da mesma forma, a potência máxima quando avaliada à velocidade de 1,57 rads. $\mathrm{s}^{-1}$ não se correlaciona de forma significativa com nenhum dos outros testes. A força de associação entre as medidas dos testes V15, V35 e AGI revelou ser moderadamente elevada a elevada ( $r=0,60$ a 0,86). Para al ém disto, a MP dos músculos extensores e flexores do joelho, avaliados à velocidade angular de 6.28 rad. $\mathrm{s}^{-1}$ mostrou correlacionar-se apenas moderadamente com a performance no V15 e no AGI. Contudo, comparando a força de associação da performance no V15 e no AGI com a MP em cada um dos grupos musculares, verifica-se um valor mais elevado com a MP dos músculos flexores do joelho.

\section{DISCUSSÃO}

As acções de alta intensidade podem influenciar positivamente a performance em futebol e ser classificadas como acções que requerem velocidade máxima, aceleração ou agilidade (10).

Genericamente, a performance de vel ocidade no presente estudo é de nível semel hante à reportada na literatura para futebolistas de el ite (para ref.'s ver 14). $O$ valor médio dos tempos no teste V 15 é similar ao observado por (2) em futebolistas profissionais ingleses. Porém, não conhecemos nenhum estudo em que a velocidade de futebolistas tenha sido avaliada sobre a distância de 35m. De facto, na maioria dos estudos as distâncias utilizadas são $10 \mathrm{~m}, 20 \mathrm{~m}, 30 \mathrm{~m}$ e $40 \mathrm{~m}$. Contudo, se analisarmos a performance através do cál culo da velocidade média para percorrer a distância de um metro (m.s-1) constatamos que os resultados no teste $V 35$ são idênticos aos descritos noutros estudos em que as distâncias de teste utilizadas foram $30 \mathrm{~m}$ e $40 \mathrm{~m}(2,3,7,9,17)$.

Relativamente aos resultados da AGI e da MP calculada a partir da avaliação da força em dinamómetro isocinético, não encontramos na literatura qualquer valor de referência para comparação.

A análise dos resultados do presente estudo permite verificar a existência de uma grande amplitude de valores nos resultados dos testes de velocidade e de agilidade (ver Quadro 1). Os resultados evidenciam uma enorme variação da capacidade física, apesar de todos os sujeitos da amostra pertencerem ao mesmo 
nível competitivo (jogadores de elite). Usando como exemplo o teste de agilidade, a diferença entre o pior e o melhor tempo foi de um segundo (18\%). Esta diferença entre tempos no teste de agilidade $(20 \mathrm{~m})$, pode traduzir-se numa distância de atraso do atleta que fez o pior tempo, em relação ao que fez o melhor tempo, de quase $4 \mathrm{~m}$; distância suficiente para que este último possa ganhar vantagem posicional numa qualquer acção de jogo.

Num trabal ho sobre a detecção de talentos no futebol, em que se aplicou uma bateria de testes físicos a jovens futebolistas, a agilidade revelou-se como o factor de discriminação mais potente entre futebolistas de elite e de sub-elite (13).

A análise dos resultados permitiu verificar a existência de uma forte associação entre a performance na $\mathrm{V} 15$ e na $\mathrm{AGI}$.

A forte associação encontrada entre os testes V15 e AGI deverá estar relacionada com o facto destes testes partilharem alguns factores comuns, como a amplitude de passada, a frequência de passada e a potência muscular. Por outro lado, os movimentos exigi dos nestes dois testes são frequentemente real izados em jogo e muitas vezes de forma combinada. Estudos do tipo tempo movimento mostraram que $85 \%$ dos sprintes realizados pelos futebolistas no jogo envolvem distâncias inferiores a $20 \mathrm{~m}$, grande parte dos quais associados a mudanças de direcção e/ ou sentido na corrida (12).

Apesar de significativa, a correlação entre a V35 com a V15 e com a AGI é menor do que a encontrada entre estes dois últimos testes. A explicação para este resultado poderá residir na maior diferença das exigências funcionais entre a $V 35$ e a AGI do que entre a $\mathrm{V} 15$ e a AGI. Adicionalmente é possível que a distância de $35 \mathrm{~m}$ seja muito longa para a avaliação de futebolistas, uma vez que apenas $5 \%$ dos sprintes realizados no jogo ocorrem sobre distâncias iguais ou superiores.

Foquemos agora a nossa atenção sobre as associações encontradas entre os resultados no V15, V35 e AGI e os resultados relativos à força muscular avaliada no aparelho isocinético.

$\mathrm{Na}$ velocidade angular de 1.57 rad.s $^{-1}$ a MP não se associou de forma significativa com nenhum dos testes de terreno realizados (V15, V35 e AGI). Este resultado estará provavelmente relacionado com as diferentes velocidades de movimento exigidas no teste isocinético e nos testes de terreno. Com efeito, resultados de diferentes estudos revelaram que a força e a MP avaliadas em dinamómetros isociné ticos a velocidades angulares relativamente baixas não apresentam val ores el evados de associação com a performance em exercícios explosivos, como os sprintes em distâncias curtas (para ref.'s ver 6). Assim, na avaliação da potência devemos atender às características dos movimentos específicos da performance na modalidade, sob pena de os resultados da avaliação não reflectirem a capacidade real dos atletas (para ref's ver 4).

Porém, na avaliação à velocidade angular de 6.28 rad. $s^{-1}$, foi encontrada associação entre a MP e a V15 e a AGI. Estes dados revelam que as exigências musculares no teste isocinético realizado numa velocidade angular mais alta estarão mais próximas das exigências funcionais dos testes V15 e AGI. Nestes testes a aceleração do movimento adquire importância fundamental, pelo que são exigidos níveis elevados de potência muscular. (19) avaliaram jogadores de futebol, basquetebol, futebol australiano e ténis em diferentes testes de força concêntrica (em dinamómetro isocinético) e em testes de velocidade numa distância de $8 \mathrm{~m}$ com mudanças de direcção em diferentes ângulos (20-60). Um dos resultados mais relevantes deste estudo foi a constatação de que o teste com maior número de mudanças de direcção (quatro) foi o aquele que apresentou valores de correlação mais elevados com as medidas de potência muscular.

A V35 não revelou associar-se de forma significativa com a MP, independentemente da velocidade angular utilizada. Durante uma parte considerável do teste de V35 o atleta realiza movimentos mais rápidos do que no $\mathrm{V} 15$ e no $\mathrm{AGl}$ e a velocidade de movimentos que exige difere muito das velocidades angulares em que os testes isocinéticos foram realizados.

Estes resultados colocam em evidência a importância da potência muscular para a realização de sprintes em distâncias curtas e/ ou com mudanças de direcção, o que não parece verificar-se quando se consideram maiores distâncias de deslocamento. Por outro lado, a velocidade angular seleccionada na aval iação com recurso a dinamómetros isocinéticos não é negligenciável na avaliação da MP de futebolistas. 
Deste dado decorre a necessidade de avaliar a MP dos futebolistas a velocidades angulares elevadas, portanto mais específicas.

A MP dos músculos flexores do joelho, avaliada na velocidade angular de 6.28 rad.s $\mathrm{s}^{-1}$, apresentou uma associação mais forte com a os resultados dos testes V15 e AGI do que a MP dos extensores do joelho. Os músculos isquiotibiais desempenham um papel importante na performance em sprinte ( para ref.'s ver 6 ).

Os músculos com maior participação na propulsão horizontal nos sprintes são os isquiotibiais, os glúteos máximos e os longos adutores. De entre estes grupos musculares, os isquiotibiais constituem o grupo muscular com a mais importante contribuição para a produção de elevados níveis de velocidade. No sprinte, os isquiotibiais, como músculos biarticulares, actuam como extensores da coxa e do joelho, organizando exactamente o movimento - concretamente, sincronizando a extensão da coxa e do joeIho. Os isquiotibiais, conjuntamente com os glúteos máximos e com os longos adutores, são responsáveis pelo movimento rápido de balanço para baixo e para trás da perna, desde o momento em que esta se encontra no ponto mais alto de elevação do joelho até à parte final da fase de apoio do pé no solo $(6,16)$. A importância dos músculos isquiotibiais para a realização de sprintes de curta distância foi também já descrita em futebolistas. Num trabalho de (8), realizado com jogadores de futebol australiano, verificouse que os tempos obtidos pelos futebolistas em testes de velocidade aos $5 \mathrm{~m}$ e aos $10 \mathrm{~m}$ se revelavam significativamente associados à força dos músculos isquiotibiais, aval iada em dinamómetro isocinético, nas velocidades de 4.19 e $6.28 \mathrm{rad}^{\mathrm{s}} \mathrm{s}^{-1}$

Concluindo, e tomando em consideração os resultados do presente estudo, sugere-se que a MP é fundamental para o desempenho nos testes V15 e AGI. Contudo, a determinação da MP em dinamómetro isocinético deve ser obtida a velocidades angulares não inferiores a 6.28 rad.s $s^{-1}$ Finalmente, embora os testes não se substituam uns aos outros, os testes V15 e AGI estarão mais próximos nos atributos que cada um deles avalia.

\section{CORRESPONDÊNCIA}

\section{António Rebelo}

Faculdade de Desporto

Universidade do Porto

Rua Dr. Plácido Costa, 91

4200-450 Porto

Portugal 


\section{REFERÊNCIAS}

1. Balsom $P$ (1994). Evaluation of physical performance. In B Ekblom, Footbal (Soccer) - $\mathrm{H}$ andbook of Sports M edicine and Science, Oxford: Blackwell Scientific Publications,

2. Brewer J, Davis J (1992). A psysiological comparison of English professional soccer players. J Sports Sci 10: 146147

3. Cometti G, Maffiuletti NA, Pousson M, Chatard JC, Maffulli N (2001). Isokinetic strength and anaerobic power of elite, subelite and amateur French soccer players. Int J Sports Med 22 (1): 45-51

4. Cronin J, McNair P, Marshall R (2001). Velocity speficity, combination training and sport specific tasks. Journal of Science and M edecine in Sport 4 (2): 168-178

5. Dawson B (2003). Speed, agility and quickness in football. In Proceedings of $\mathrm{V}$ W orld Congress on Science and Football. Lisbon-Portugal. p 14.

6. Delecluse C (1997). Influence of strength training on sprint running performance. Sports M ed. 24 (3): 147-156

7. Dupont G, Akakpo K, Berthoin S (2004). The effect of inseason, high-intensity interval training in soccer players. J Strength Cond Res 18 (3): 584-589

8. Hrysomallis C, Koski R, McCoy M, Wrigley T (1999). Correlations between field and laboratoty tests of strength, power and muscular endurance for elite australian rules footballers. In Proceedings of Fourth W orld Congress on Science and Football. Sidney, Australia. p 81-85.

9. Kollath E, Quade K (1991). Measurement of sprinting speed of professional and amateur soccer players. In Proceedings of Second W orld Congress of Science and Football. Eindhoven. $p$

10. Little T, Williams A (2005). Specificity of acceleration, maximum speed, and agility in professional soccer players. J Strength Cond Res 19 (1): 76-78
11. Pauole K, Madole K, Garhammer J, Lacourse M, Rozenek $R$ (2000). Reliability and validity of the T-Test as a measure of agility, leg power, and leg speed in college-aged men and women. J Strength Cond Res 14 (4): 443-450

12. Rebelo A (1993). Actividade física do futebolista em competição. In Provas de Aptidão Pedagógica e de Capacidade Científica. Instituto Superior de Educação Física Universidade do Porto, Porto.

13. Reilly T, Williams AM, Nevill A, Franks A (2000). A multidisciplinary approach to talent identification in soccer. J Sports Sci 18 (9): 695-702

14. Stolen T, Chamari K, Castagna C, Wisloff U (2005). Physiology of soccer: an update. Sports M ed 35 (6): 501-536

15. Weyand PG, Sternlight DB, Bellizzi MJ, Wright S (2000). Faster top running speeds are achieved with greater ground forces not more rapid leg movements. J A ppl Physiol 89 (5): 1991-1999

16. Wiemann T, Tidow G (1995). Relative activity of hip and knee extensores in sprinting - inplications for training. New Studies in Athletics 10 (1): 29-49

17. Wisloff U, Castagna C, Helgerud J, Jones R, Hoff J (2004). Strong correlation of maximal squat strength with sprint performance and vertical jump height in elite soccer players. Br J Sports M ed 38 (3): 285-288

18. Wrigley T, Strauss G (2000). Strength assessment by isokinetic dynamometry. In CJ Gore, Physiological tests for elite athletes, Champaign, Illinois: Human Kinetics, 155-199

19. Young W, James R, Montgomery I (2002). Is muscle power related to running speed with changes of direction ? J Sports M ed Phys Fitness 42 282-288

20. Young W, McDowell M, Scarlett B (2001). Specificity of sprint and agility training methods. J Strength Cond Res 15 (3) : 315-319 